\title{
Shelf System Localization Algorithm Research based on RFID Technology
}

\author{
Li Zhü ${ }^{1, \text { a }}$, Jian Lan Liü,b \\ ${ }^{1}$ College of Mathematics and Computer Science, Jiangxi Science and Technology Normal \\ University, Nanchang, China \\ ${ }^{2}$ College of Mathematics and Computer Science, Jiangxi Science and Technology Normal \\ University, Nanchang, China \\ abailan_1982@sina.com, ${ }^{b}$ ljlan0821@163.com
}

Keywords: Localization algorithm; RFID technology; Smart shelves; Reference label; Database

\begin{abstract}
With the rapid development of information technology, radio frequency technology application has become an important symbol of intelligence realization. According to the requirements of intelligent bookshelf system, this paper analyzes and designs intelligent bookshelf system structure from two aspects of hardware and software. In determining positioning algorithm of intelligent bookshelf system, analyze the classification of indoor location algorithm and advantages and disadvantages of various algorithms, select RFID indoor localization algorithm because of intelligent bookshelf system's actual demand for location selection, and improve it on the basis of LANDMARC system algorithm. Analyze from received power selection, reference label distribution, nearest neighbors number set and algorithm weight set and so on, design books accurate localization algorithm scheme, and make experiments to test the experimental data and algorithm validation to effectively improve smart shelves system book positioning accuracy.
\end{abstract}

\section{INTRODUCTION}

Library is the important place for us to obtain knowledge. Only with constantly updated knowledge can we keep up with the pace of The Times. Book collection number and categories changing make the library work under pressure: people have a choice of targeted reading, and at the same time, the large number of books has increased the difficulty of library managers and readers query and retrieve books. Wireless radio frequency identification technology is increasingly mature which widens the scope of its application. This technology can be applied in library management to greatly enhance service quality and improve work efficiency.

\section{SMART SHELVES SYSTEM}

RFID technology is a kind of non-contact automatic identification technology based on wireless communication technology. It automatically identifies target object through radio frequency signal transmission and accesses to relevant data and provides it to background computer processing system for corresponding data processing [1]; Smart shelves system is a kind system equipped with RFID readers, RFID label and RFID transceiver antennas. Reader can detect and locate the actual location shelf of the books at any time, and it is a set of intelligent and digital books management system management system for real-time books and location data information processing and book position location by reader and computer connection [2].

Library intelligent bookshelf system mainly has four participating entities: readers, intelligent bookshelf ontology, librarians and library literature to facilitate part entity management operation, maximally improve the performance of intelligent bookshelf system, and improve the performance of the system[3]. Intelligent bookshelf system entity relationship diagram is shown in Figure 1: 


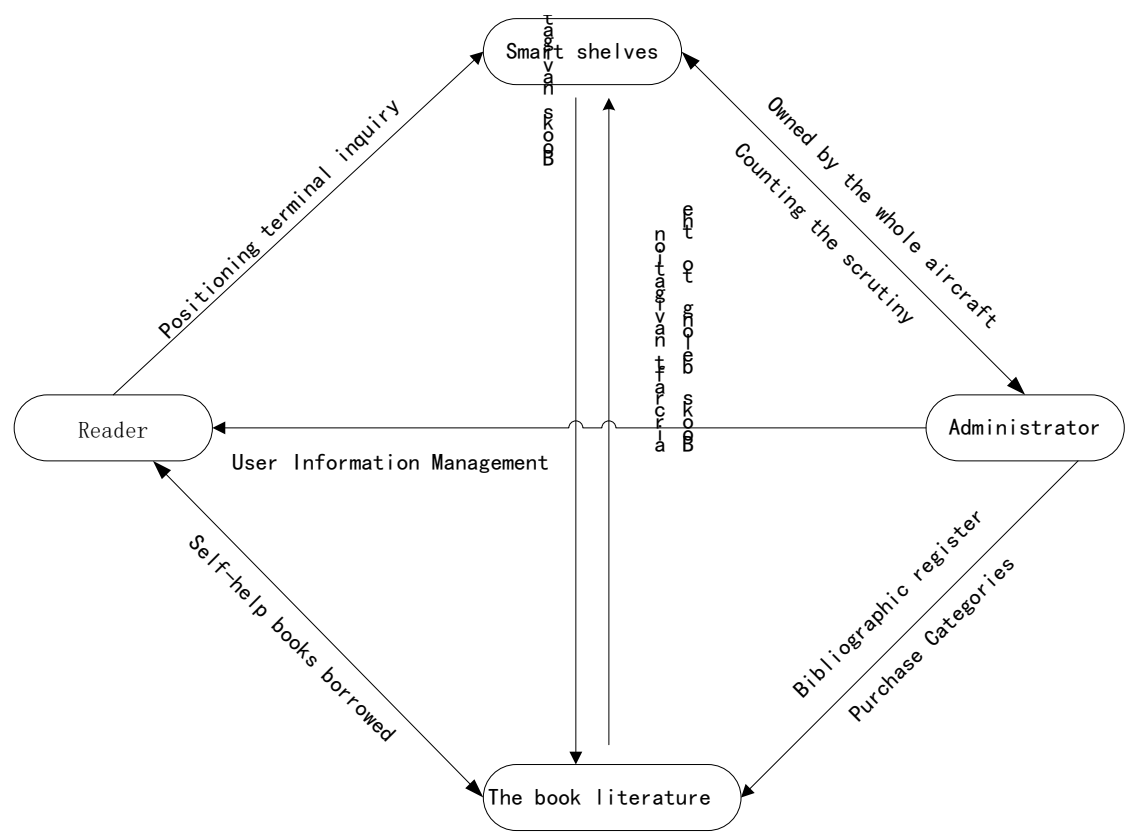

Figure 1 System entity relationship diagram

One of important steps of system analysis and design is to build a system use case model. System use case is to make functional description of system from the angle of the user, and describe the connection between system and system participants.

\section{INTELLIGENT BOOKSHELF SYSTEM LOCALIZATION ALGORITHM}

According to the different way of positioning, RFID indoor localization algorithm is divided into three categories: signal arrival time localization algorithm, signal arrival angle positioning algorithm and received signal strength positioning algorithm [4].

A. Signal arrival time localization algorithm

Signal arrival time is TOA. The localization algorithm uses moving targets and measures range wireless signal propagation time to determine the location, which is to determine the location of the object by measuring travel time or the time difference of electromagnetic wave from launcher propagation to multiple receivers' devices [5]. Assume signal propagation time between the moving target and base station is $t$, then the distance between them can be represented with $r=v t$. Because RF signal speed value in the air is almost Guangsu C, use the following formula to calculate the distance between the moving targets to the measure device:

$$
\mathrm{r}_{\mathrm{i}}=\mathrm{c} \cdot\left(\mathrm{t}_{\mathrm{i}}-\mathrm{t}_{0}\right)
$$

Figure 2 is TOA algorithm diagram.
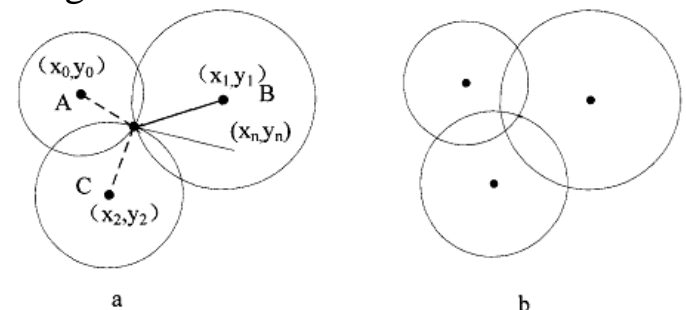

b

Figure 2 TOA algorithm diagram (a is the bit of three circles interaction, $\mathrm{b}$ is the multipoint of three circles interaction)

By moving three measuring devices closer to the target distance, get three distance values and the moving target position coordinates can be calculated. By the nature and formula of circle e $=\sqrt{\left(x-x_{0}\right)^{2}+\left(y-y_{0}\right)^{2}}$, solve $\left(x_{n}, y_{n}\right)$ coordinates with the simultaneous equations obtained; In actual situation, located region may not interact to one point, as is shown in Figure $2 \mathrm{~b}$, and TOA algorithm has extremely high requirements for the synchronization of transmitters and receivers. There is a need to add time identification to the signal and positioning device points should be at 
least three or more, so this will inevitably cause some errors; To improve the positioning precision, other algorithms should be introduced for position compensation, but the system cost and the complexity of algorithm also increase, so TOA is not suitable for the conditions of intelligent bookshelf system positioning.

\section{B. Signal arrive angle positioning algorithm}

Wireless radio frequency signal launched by the moving target is measured by antenna array. Receiving device measures electromagnetic wave incident angle and forms a location line from the launcher to receiver to determine the direction of the moving target location and implement positioning according to the signal arrive angle. This is AOA location [6]. The advantage of signal arrive angle positioning algorithm is that it only needs two reference points to determine the target location, and there will be no positioning fuzzy phenomenon with multiple interaction points in the movement path; But AOA location has a high requirement on the hardware. Hardware equipment is complex and precision is greatly affected by environment multipath effect, so, in order to measure incident angle accurately, not only improve receiver antenna, also install directional antenna array.

\section{Received signal strength positioning algorithm}

Typical positioning algorithm application systems based on receiving information strength are RADAR system and LANDMARC system [7]; RADAR system is the earliest use of wireless radio frequency signal receiving strength RSS to develop RFID indoor positioning system. This approach has application limitations, and is mainly applied in two situations: one is the need to combine with other relative positioning technologies to improve the accuracy of positioning; The second is applied to not high user requirements for positioning accuracy, such as the movement of the target range, etc.; RADAR positioning principle is very simple, and its system model can be calculated by experimental data, and can be derived through experience theory; With combination of experience system testing and signal transmission model, it requires less information reference points, and the bottom wireless network structure design is simple and convenient for system installation; Compared with TOA algorithm, the localization algorithm based on RSS is less affected by the indoor environment multipath effect, but in indoor environment, radio frequency signal propagation model is complex, RADAR system positioning accuracy based on RSS is low.

LANDMARC system is based on active RFID electronic label signal strength's indoor location identification system. It uses reference label as the system's reference positioning point for auxiliary orientation, and estimate distance by obtaining signal strength value of reference labels and labels under test to real-time forecast the location of the object under test [8]. Locating method is the commonly used measuring positioning way at present for indoor positioning. System pastes reference label in indoor needs to locate the position of the known coordinates and records electromagnetic wave signal intensity launched by reference label with readers in a fixed position. When the under test target label gets to positioning radiation area, reader detects the energy value sent by the target label. It also is the comparison of received signal strength value and the RSS value sent by the recorded reference labels. Through a certain location coordinates calculation, determine the position information of the under test target.

\section{EXPERIMENTAL SIMULATION ANALYSIS}

\section{A. RFID indoor positioning principle}

RFID indoor location improved the technology of LANDMARC system, and improved the positioning accuracy by changing reader distribution and quantity, adopting passive labels, determining reference label distribution and quantity, and improving algorithm process in order to suit the positioning requirements of library environment intelligent bookshelf system; A basic wireless radio frequency positioning system usually consists of three parts: data reading acquisition device, location estimation localization algorithm and front end position display application program [9]. RFID indoor positioning system's working principle is: first, the user issues the locate request, and the system sends request and submits it to the reader. The reader sends radio frequency signals by radiation power to activate electronic labels. Labels return memory information or signal strength information through radio frequency signal to reader, and then select localization algorithm. 
Make position estimation through the signal information of different measuring time, angle, and received signal strength of TOA and AOA, RSS algorithms. Calculate the under test target location coordinates with neural network, nearest neighbors algorithm, probability calculation method, and with transformation use display system for users to read.

$B$. The setting of localization algorithm parameters

(1) Reference label distribution

Pending label location is finally located based on several references label position distributed around it, so reference label distribution method will affect the accuracy of the algorithm. The intelligent bookshelf system positioning is accurate to bookshelf minimum cell. Take a minimum cell of the shelf as the research object, and then expand the whole bookshelf. Assume evaluation position precision parameters are the average position error, and the expression can be expressed as:

$$
M E E=\frac{1}{M} \sum^{M} \sqrt{\left(x-x_{0}\right)^{2}+\left(y-y_{0}\right)^{2}}
$$

The actual location coordinates of the under test label is (x0, y0). Localization algorithm is used to get the estimation coordinates (x,y). $\mathrm{M}$ is the number of experimental simulation. Take second order weight as the weight for respective simulation, and the average position error got is shown in Table 1.

Table 1 Different reference label number average position error table

\begin{tabular}{|l|l|l|l|}
\hline $\begin{array}{l}\text { Reference } \\
\text { label } \\
\text { number }\end{array}$ & $\begin{array}{l}\text { Average position error } \\
/ \mathrm{m} \mathrm{\quad (100} \mathrm{times)}\end{array}$ & $\begin{array}{l}\text { Reference } \\
\text { label } \\
\text { number }\end{array}$ & $\begin{array}{l}\text { Average position } \\
\text { error/m (100 times })\end{array}$ \\
\hline 6 & 0.0650 & 12 & 0.0432 \\
\hline 7 & 0.0551 & 13 & 0.0525 \\
\hline 8 & 0.0335 & 14 & 0.0610 \\
\hline 9 & 0.0495 & 15 & 0.0650 \\
\hline 10 & 0.0526 & 16 & 0.0687 \\
\hline 11 & 0.0455 & & \\
\hline
\end{tabular}

Figure 3 is error distribution for different label number.

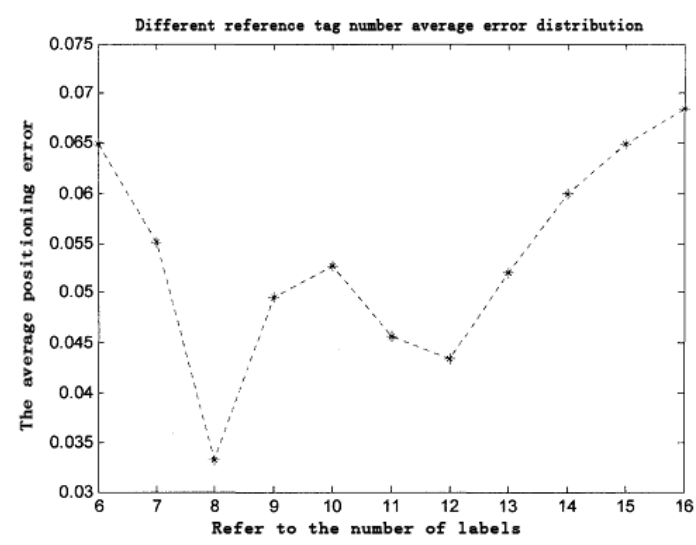

Figure 3 Different reference label number error distribution

Through simulation analysis, when the reference label number is 8 , there is the smallest average position error. The average orientation error of 100 times is $0.0332 \mathrm{~m}$, so paste a reference label on bookshelf minimum cell's eight apex angles, and two adjacent cells use four reference labels on the connection surface.

(2) The nearest neighbors label number set

The number of nearest neighbors label decides the number of reference labels involved in calculating in system algorithms, which can significantly affect the coordinates of the target label under test. The number of nearest neighbors' labels can be one, two or more. These label coordinate values eventually determine the position of the target label according to certain weight proportion sum. This algorithm is called KNN nearest neighbor algorithm; larger error reference labels may bring greater error when positioning coordinates calculation weight in order to improve the position 
precision of the system algorithm, so consider the determination of nearest neighbors label number $\mathrm{k}$ value in demand analysis. According to under test label signal strength value and reference label signal strength value, obtain Euclidean distance vector $E$ to regulation sequence. Make weighted the minimum Euclidean distance reference label position coordinates with under test label. Experimental results show that if $\mathrm{k}$ value is too large or too small, it was not availed to improve the precision of positioning, so determine the size of the $\mathrm{k}$ value through specific experiments.

Through data analysis, when the number of nearest neighbors' labels is 8 , average position error amount is the minimum. The average error of 100 times orientation is $0.0239 \mathrm{~m}$, and $\mathrm{k}$ value is 8 to facilitate delimit the smallest scope. Limit the label under test within the smallest cell of first layer of the shelf. This improves the existing intelligent bookshelf which only limits fault plane books in one layer and improves the positioning precision.

(3) Determine the nearest neighbors label weights

There are many ways to determine the nearest neighbors label weights, but they must satisfy two basic conditions: ownership weights weightings sum must be 1 , which is accord with $\mathrm{W} 1+\mathrm{W} 2+\ldots+\mathrm{Wk}=1$; weight $\mathrm{W}$ must be monotony decrease function, namely if reference labels and the Euclidean distance $\mathrm{E}$ of labels under test are larger, weight $\mathrm{W}$ of reference labels must be smaller. Commonly used ways of weight setting are one order weights value, second order weights value and right logarithmic weights value; target label location values got by different weights setting methods are not the same. In order to achieve high precision positioning effect, analyze the influence of different weights setting methods on label location coordinates.

First define a variable to describe the position and signal intensity of under test label, calculate close degree of a minimum $\mathrm{E}$ value reference label with Euclidean distance. So single label dependent factor is $\xi$, and the expression is as shown in Formula (3).

$$
\xi=\left(W_{i}\right)_{\max }
$$

When the under test label signal characterization distance $E_{i}$ is of the same value, the bigger label dependent factor $\xi$ shows the greater single label dependence, that is to say that the calculated under test label position is closer to the largest weight and the nearest neighboring reference label position. By analyzing, it is known that quadratic weighting setting method's single label dependence is on the highest degree. This means that label location coordinates are closer to the nearest neighbors coordinates, and logarithmic weighting setting method has the lowest single label dependence. The localization algorithm adopts quadratic weighting weights; each reference label has a weight $\mathrm{Wi}$, and the bigger weights means that it is the closer to the under test label.

C. Analysis of experimental results

Experiments are done to validate the effectiveness of intelligent bookshelf localization algorithm. Locate within four adjacent minimum cells of two sides of intelligent bookshelf first floor in the library. Select the minimum cell size as $1.0 \mathrm{~m} * 0.5 \mathrm{~m} * 0.5 \mathrm{~m}$, and paste eight reference labels respectively on eight cell vertex angles of the minimum cell. Publically use four labels at adjacent two units' joints. Without considering spatial coordinates in the vertical direction, describe the under test coordinates value, which is shown in Table 2:

Table 2 Under test label's actual coordinates

\begin{tabular}{|c|c|c|c|}
\hline Under test label & Coordinates & Under test label & Coordinates \\
\hline 1 & $(0.25,0.25)$ & 5 & $(0.72,0.75)$ \\
\hline 2 & $(0.50,0.30)$ & 6 & $(1.25,0.63)$ \\
\hline 3 & $(1.05,0.25)$ & 7 & $(1.68,0.80)$ \\
\hline 4 & $(0.45,0.80)$ & 8 & $(1.75,0.90)$ \\
\hline
\end{tabular}

Under test label estimation position coordinates calculated by algorithm simulation is as shown in Table 3: 
Table 3 Under test label estimation position coordinates

\begin{tabular}{|c|c|c|c|}
\hline Under test label & Real coordinates & Under test label & Real coordinates \\
\hline 1 & $(0.32,0.22)$ & 5 & $(0.70,0.72)$ \\
\hline 2 & $(0.47,0.28)$ & 6 & $(1.24,0.63)$ \\
\hline 3 & $(1.03,0.21)$ & 7 & $(1.64,0.79)$ \\
\hline 4 & $(0.48,0.82)$ & 8 & $(1.77,0.93)$ \\
\hline
\end{tabular}

The average position error of under test label obtained by calculation is $0.0236 \mathrm{~m}$. Intelligent shelves localization algorithm gets higher positioning accuracy and satisfies the readers demand to locate books to minimal cells.

\section{Conclusion}

Wireless radio frequency identification technology is a new positioning information acquisition and processing technology, which is widely used in logistics management, retail and other various fields. In order to improve the efficiency of reader's borrowing and librarians' work efficiency and realize intelligent management and improve library service level. Compared with the existing intelligent bookshelf, intelligent bookshelf system positioning precision algorithm has greatly improved according to library environment and reader position needs. From books locating shelf one floor to bookshelf minimum cell, it reduces the complexity of the system, and reduces the system cost. It also can effectively simplify books borrowed process and improve the automatic identification and rapid positioning ability of books.

\section{REFERENCE}

[1] Li Weifeng, Wang Dong. Indoor location technology research based on RFID [D]. Shanghai: Shanghai Jiaotong University, 2010

[2] Tan Zhongding. Rfid tracking technology libraries disorderly frame strategy comparison [D]. Journal of Guangxi Academy of Sciences, 2011

[3] Chen Congchan, He Lianglun. Regional refinement of RFID indoor localization algorithm [J]. Journal of Computer Applications and Software, 2011

[4] Chen Bing, Liu Kaihua, Shi Weiguang. Virtual label improved algorithm based on RFID [J]. Computer Engineering, 2011

[5] Yao Yuan. The application research of RFID technology in university library [D]. Shanghai: Shanghai Jiaotong University, 2011

[6] Zhao Jingliang, Yao Jinjie, Su Xinyan. Indoor positioning system design based on radio frequency identification [J]. Computer Measurement and Control, 2011

[7] Tan Hengya. Insanity open-shelf books borrowing and reading phenomenon countermeasures in university library [J]. China's Economy and Trade, 2010

[8] Jiang He, Gao Hui. The library information management system based on RFID [D]. Chengdu: University of Electronic Science and Technology, 2010

[9] Zhang Yusong, Du Jinglin. Library intelligent bookshelf scheme research and design based on uhf RFID. Nanjing Information Engineering University Library. Nanjing. Library Journal. 2014

[10] Chen Wei, Shen Zeqian. Data visualization [M]. Beijing: Electronic Industry Press, 2013

[11] Ma Xiaoting. Library data visualization analysis system design and implementation. Lanzhou Information Engineering College, 2015 